\title{
Rules for an Ontology-based Approach to Adaptation
}

\author{
Thanh Tran, Philipp Cimiano, Anupriya Ankolekar \\ Institute AIFB, University of Karlsruhe \\ \{dtr,pci,aan\}@aifb.uni-karlsruhe.de
}

\begin{abstract}
Adaptation addresses the complexity and overload caused by an increasing amount of available resources by the identification and presentation of relevant resources. Drawbacks of existing approaches to this end include the limited degree of customization, difficulties in the acquisition of model information and the lack of control and transparency of the system's adaptive behavior. This paper proposes an approach which addresses these drawbacks to perform meaningful and effective adaptation by the application of semantic web technologies. This comprises the use of an ontology and adaptation rules for knowledge representation and inference engines for reasoning. The focus of this paper lies in the presentation of adaptation rules.
\end{abstract}

\section{Introduction}

Due to the increasing amount of available informational as well as transactional resources, most prominently on the Internet, it is desirable to develop systems that can filter this large resource space to a smaller set that is more relevant (to the user). Whereas the corresponding process is called adaptation - or personalization when geared towards the user applications with this support are referred to as adaptive systems.

Existing approaches to adaptation can be distinguished along different criteria: the aspects that are represented (adaptivity dimensions), how they are represented (representation formalism) and how the models are used to perform adaptation (exploitation techniques).

With respect to the adaptivity dimensions, collaborative filtering [1] and content-based filtering [2] make use of user-related and content-related information, respectively, to find out what is "relevant". Due to limited customization of resources to the user and other problems such as "new-item", "sparsity" and "over-specialization" [3], these dimensions are combined [2] and extended to capture possible presentations of the content [4], possible structures of the content in terms of narrative models [5], the user task [6], the system and the environment. In fact, it has even been proposed to make the adaptation logic more explicit in the form of an adaptation model [7].

Popular formalisms for the representation of these different aspects include vectors, matrices, weighted ngrams, decision trees, artificial neural networks, Bayesian networks and weighted associative networks. Correspondingly, techniques for the exploitation of the resulting models are adopted from statistics and machine learning. They are used to calculate similarities and to classify users, content etc.

The various attempts to combine content- and user related information and to extend them with further adaptivity dimensions make a higher level of customization to the user and other requirements possible. However, apart from the inherent difficulties in collecting, and especially, in exchanging model information, these approaches have been criticized to be complex, computerized oracles, which give advice but cannot be questioned [8]. The reasons for recommendations cannot be explained to the user due to the complexity and nature of the underlying algorithms that employ latent factors and heuristics.

Therefore, we leverage strengths of existing approaches by the incorporation of all the dimensions that have been identified as relevant for adaptation. The drawbacks are addressed by the use of semantic web technologies. Whereas ontologies are used to increase interoperability and reusability of model information, rules are employed to represent the adaptation logic in a way that users can inspect, understand, and even modify the rationales behind adaptive functionalities. In section 2 , we begin with an overview of the approach. Then, in section 3, we briefly present some ontology concepts important for the understanding of adaptation rules, which will be discussed in details in section 4. Finally, we present similar work (section 5), conclusion, evaluation, open issues and on-going work (section 6). 


\section{Overview}

We illustrate the main ideas of this approach on the basis of a personalized portal as shown in Figure 1. This is an extension of the Liferay portal architecture encompassing four functional modules. Apart from basic navigation and search, the system is able to track user interactions, generate recommendations in the form of links and present the content chosen by the user. Figure 1 shows a user reading an Introduction about OWL. This establishes a context on which adaptation takes place. As shown, recommendations to this include related content units, which among others, e.g. OWL semantics and RDF, have also OWL as subject.

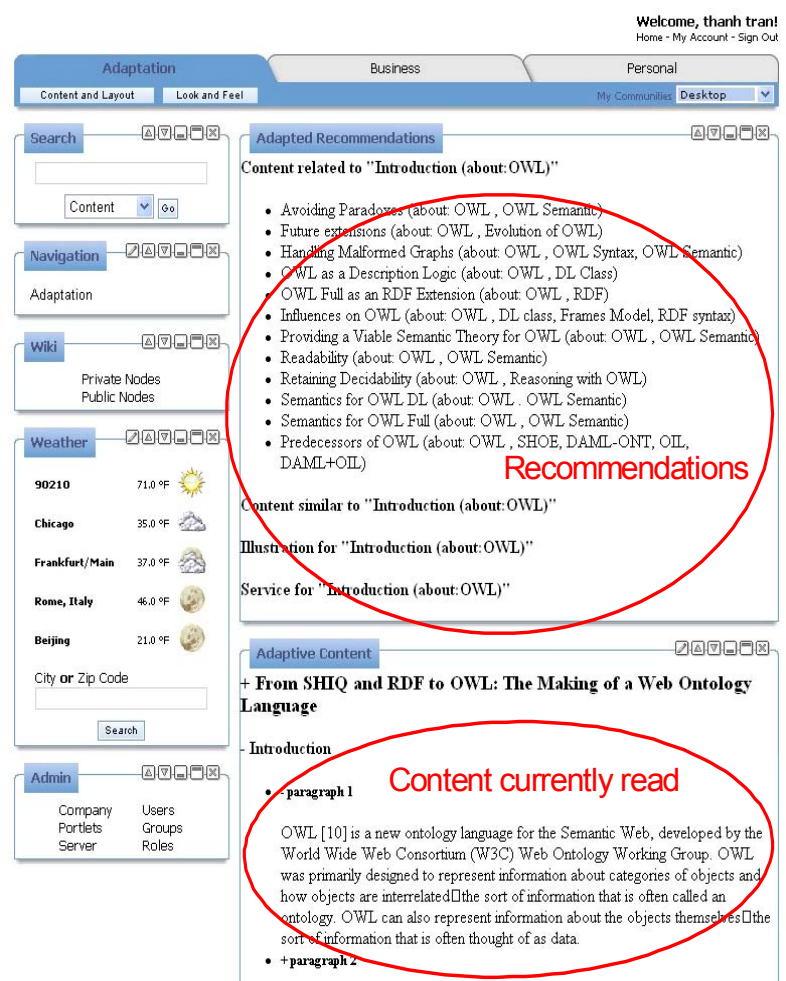

Figure 1 - A personalized portal

Such recommendations based on semantic relatedness are possible by the annotation of each paragraph such as Introduction in the example, with the entities that it deals with. In general, we employ a fullfledged ontology-based approach to adaptation. We have developed an ontology for the domain of adaptive systems (ODAS) to represent all the adaptivity dimensions employed by existing approaches. ODAS comprises of concepts and relations to talk about content, user, task, environment etc. With respect to the above example, Introduction is an instance of the ODAS concept Content about Entity. OWL and the other entities described by this and by recommended content units mentioned above belong to an additional domain ontology that is employed to represent entities the content resources refer to. Note that the ontologybased representation of model information facilitates reuse and exchange because language constructs, i.e. axioms, can be used to limit possible (mis-) interpretations of the concept semantics. Thus, the problem with model acquisition can be alleviated by the ontology-based reuse and exchange of information - content metadata in particular - between systems.

ODAS concepts and relations are then used in adaptation rules, which represent and perform adaptation logics in a manner transparent to the user. Besides, they can be made editable to increase user control, an HCI aspect important for usability that is not supported in current computerized oracles. The recommendations shown above, for instance, are results of the firing of a rule. This rule exploits the relatedness of content semantics to perform adaptation.

\section{ODAS - towards a Domain Ontology for Adaptive System}

ODAS, the ontology we introduce here aims to provide a conceptualization of objects and relations that are relevant for the adaptation of hypermedia resources to the user context. It corresponds to the definition of ontology proposed in [9]: entity types are explicitly and formally defined on the basis of OWL. Following design principles and guidelines such as minimal ontological commitment and minimal encoding bias [9], we axiomatize commonsense knowledge of the domain in a way less prone to biases and assumptions. Also, terminologies and ontologies that can be seen as being greatly agreed-upon, are incorporated - SUMO [10], OWL-S Process ontology [11], LOM [12] and PAPI [13] in particular. The objective is to accomplish a shared conceptualization of the adaptive system domain that can cater for extensibility and semantic interoperability.

Figure 2 shows a portion of the subclass hierarchy of ODAS. We briefly illustrate how the context and the various adaptivity dimensions (as highlighted by rectangles in Figure 2) can be represented by the use of this ontology. Central to the representation of the adaptation context is the notion of Process. Application Interaction for instance, tells the system that a particular User is involved (user model), and currently, is interacting with a Content resource (domain model) of the Application (system model) to accomplish a task. Indirectly, this task is captured as a Composite Process of which the atomic interaction is part of (task model). That is, we employ a process-orientated representation 
of tasks. The workflow required to accomplish the task is modeled in the system as a composite Computeraided Process. The output of this process can be implicitly assumed to be the user's goal so as to drive adaptation towards this end. Based on this semantics, rules can be designed to utilize relations, e.g. is pre and is post, among sub-activities of such a workflow to perform task-based adaptation.

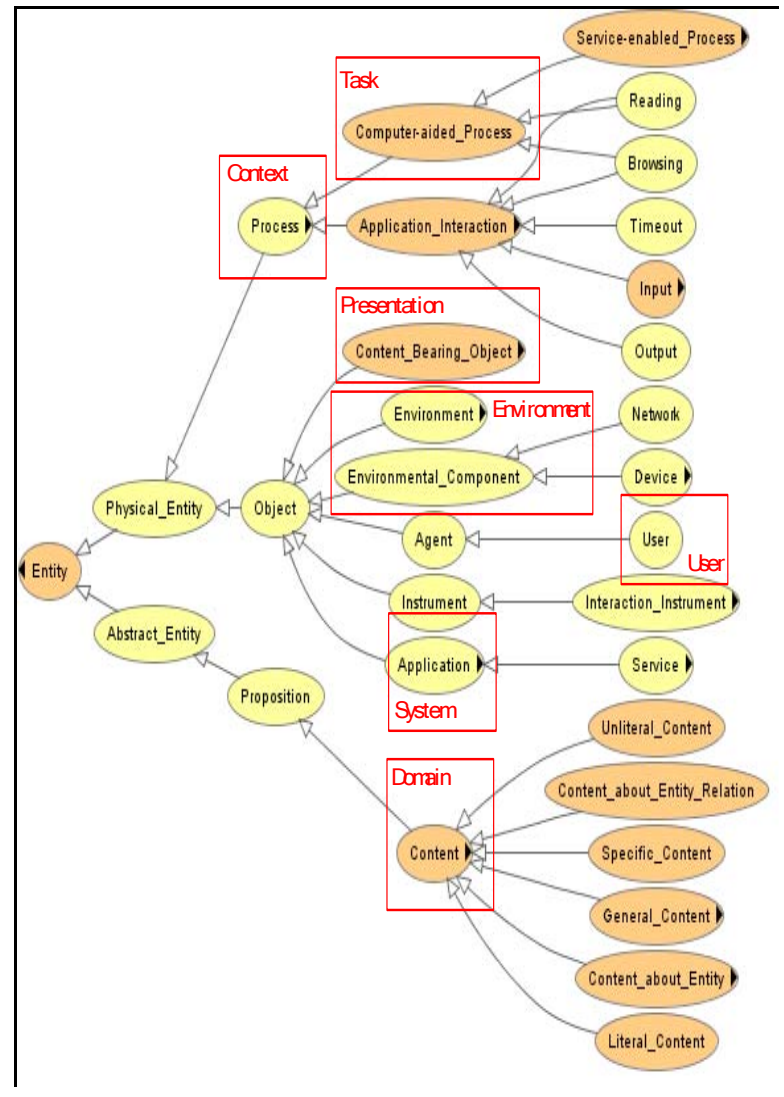

Figure 2 - Hierarchy of selected concepts

Note that recorded Application Interactions contain information about the Content currently processed by the User. Since Content types are distinguished by the subjects they describe, content-based adaptation rules can be used to trigger recommendations suggesting users to navigate to related content units of different types. A special type of Content is Executable Content, which differs from others in that it is embodied in a UI Element and is representation of a Service. This way, any services can be indirectly represented as individuals of Executable Content and adapted to the User in the same fashion as with other content types. Also, we introduce the concept of Content Bearing Object (CBO) to distinguish the actual materialization from the abstract Content embodied in it. This way, concepts become available for the representation of layout- and presentation-related requirements (presentation model).

Further concepts that deliver contextual information are User and Environment. User properties such as has credential, has read, knows, has interest, is able to are employed as constraints in the rule. Also, characteristics of the Environment play a similar role in adaptation (environment model). Restrictions in the Environmental Component such as bandwidth of the Network and size and resolution of the Display Device, must be considered to deliver relevant resources.

Now, we continue to show how adaptation can be designed to exploit the notion of process context and to meet the requirements and constraints as implied by such user and environmental descriptions.

\section{A Rule-based Adaptation Model}

While the ontology represents the different adaptivity dimensions in terms of user, domain, task, environment and system model, this section demonstrates that the logic underlying the adaptation can also be explicitly captured on the basis of a rulebased model. Just like the decision to use OWL for ontology modeling, we aim to increase interoperability, reusability and extensibility by the choice of SWRL, another $\mathrm{W} 3 \mathrm{C}$ recommendation for the representation of rules.

\subsection{Structure and Components of a Rule}

In general, atoms in the rule body capture the conditions that need to be fulfilled for the recommendation stated in the head to apply. As shown in Figure 3, conditions in the rule body are split up into three categories: context-related, adaptation-related and constraints-related. The first category ensures that the right context is given. This is represented by the concept of Process, which serves as the "entry point" to access various adaptivity dimensions, i.e. Content, Task, User, Application and Environment.

In the second category, the semantics of the Content concept is used to perform adaptation. Given the User is Reading a content unit, the adaptation mechanism recommends resources related to this. While this is referred to as content-based adaptation, task-based adaptation can also be applied to exploit the semantics of the Process concept. Eventually, these styles of adaptation yield a set of resources related to the one the user is currently interacting with. The last category consists of conditions acting as constraints that, when applied, have a minimizing effect on this adapted set. The example shows how user information can be used to restrict the set of related resources to a set for which 
could reflect a particular relation (dependency, causality etc.) between denoted entities - which might be only in the mind of the author and not directly encoded in the ontology. Using relations modeling the structure of the content such as has part, is pre and is post, the narrative sequence given by the author can be reproduced, e.g. by recommending resources annotated as the subsequent content of the one currently read:

Atomic_Content(?x) $\wedge$

Atomic_Content(?y) $\wedge$ is_post_content(?y,?x)

4.2.3. Task-based Adaptation. Similarly, task-based adaptation makes use of the given sequence of process execution as modeled in the workflow in order to make recommendations for services that fit with the current task. As discussed, the workflow, and indirectly also the task is modeled and represented as a Computeraided Process. Thus, when the User is involved in an interaction that is part of a Computer-aided Process, then the system recommends subsequent processes as given by the is post relation until the user accomplishes the task, i.e. obtains the output of the Computer-aided Process. More precisely, the system would recommend an Executable Content, which is a representation of the Service. This service acts as the instrument of the subsequent process:

Computer-aided_Process(?workflow) $\wedge$
Application_Interaction(?p1) $\wedge$ is_part_of(?p1, ?workflow)
$\wedge$ User(?u) $\wedge$ is_involved_in(?u,?p1) $\wedge$
Application_Interaction(?p2) $\wedge$ is_post_process(?p2,?p1) $\wedge$
Service(?s) $\wedge$ instrument(?p2?s) $\wedge$
Executable_Content(?y) $\wedge$ is_repreentation_of(?y?s)

It might also make sense to incorporate advanced semantics of relations and entities involved in Process into adaptation, i.e. semantically-related services.

4.2.4. Applying further Constraints. Having generated the numerous recommendations on the basis of different styles of adaptation, different requirements can be applied to limit them to a set of relevant resources. This would be the case if they meet users' requirements and can be appropriately presented to the user, given the environmental constraints. For instance, a Content can be seen as relevant, if the User has credential for, does not know, has not read or has interest for it (or some entities subsuming it). The instantiation of the constraints-related category may be as follows:

Content $(y) \wedge$ Credential(?c) $\wedge$ User(u)
required_credential(?y,?c) $\wedge$ has_credential(?u,?c)

When taking restrictions in resolution and size of the Display Device into consideration, only a preview version of the resources may be recommended.

\section{Related work}

As discussed in section 2, such a semantic approach can address several problems common to traditional ones. With respect to similar work, this approach is distinct in the degree of comprehensiveness and generality. Other approaches are mostly geared towards specific problems of adaptation [14][15]. The concepts introduced are not sufficient to capture the various dimensions valuable for a fine-grained adaptation. Also, whereas the domain model is represented as a set of keywords there, we explicitly introduce the notion of Content-Bearing-Object, Content and subject. The subject of a content unit refers to entities of a domain ontology. While keywords have no formal meaning per se, the semantics of entities provided by domain ontology can be exploited by a reasoner to classify Content individuals. Also, the few rules proposed can not be compared with the sets we have discussed. The generic structure discussed for adaptation rules can be instantiated in many ways to perform different styles of adaptation and to meet different constraints imposed by the user and the environment.

\section{Conclusion, Open Issues and Outlook}

We have introduced an ontology-based approach that allows for adaptation customized to different requirements. The user demand is derived from the knowledge contained in and entailed by the ontology. This is implicitly matched with the resource supply on the basis of various conditions captured in the body of SWRL rules. As a result of the firing of rules, recommendations in the form of content units are generated, which can be used to implement the concept of adapted content and adapted navigation. Likewise, adapted presentation can be achieved when layout and presentation-related conditions are incorporated into the rules by the use of the CBO concept. While this proposal cannot be considered to be complete, it has been designed to maximize extensibility by the use of de-facto standard languages, terminologies and ontology modeling principles. The ontology may serve as a foundation that can be extended and modified to arrive at the ontology for the domain of adaptive systems. Also, the rules can be modified (by the user) for specific adaptation requirements.

As a proof of concept, we have developed a personalized portal. Preliminary evaluations show that recommendations reasonably match the resources that would have been manually chosen by the user. In the approach presented, the execution of adaptation rules is decidable due to the restriction to Description Logic 
Program. Thus, the quality of recommendations depends only on the degree of correctness as to how the rules reflect the style of adaptation the user wishes. Note that the set of rules is sufficiently comprehensive and most importantly, can be controlled by the user. Thus, it is likely that this semantic approach is effective.

However, when considering the resources consumed, it is yet, rather inefficient. The current manual annotation of content units requires a lot of time. Besides, the inference engine requires several minutes to process and update recommendations. In the illustrated prototype, KAON2 is employed as the knowledge management infrastructure, supporting reasoning with and persistent storage of OWL statements as well as DL-safe rules [16]. In addition, we are working on the integration of a special purpose metadata crawling and extraction tool with this backend infrastructure. Eventually, we would like to develop an adaptive system which can automatically acquire metadata, record user interactions, prioritize and apply adaptation rules and run queries to retrieve recommendations. This is then used to assess the relative effectiveness and efficiency of the entire system against comparable approaches.

Finally, apart from open issues of automatic metadata acquisition and ontology merging, we like to point out on-going work: finding the appropriate tradeoff of semantic interoperability and efficiency for the domain of adaptive systems. This is because while OWL allows for greater expressiveness and interoperability by providing many constructs to restrict unintended interpretations, there are currently not efficient reasoners available. In fact, state-of-theart OWL reasoners such as OWLIM and KAON2 do not perform well with the combined use of disjunctions and equality as they open up drastically the space of possible solutions that have to be explored for making inferences. Therefore, we had to eliminate equality. Thus, while we still think that OWL-Full is appropriate and even required for communication and presentation, in the application, OWL-DL without number restrictions and nominals has been used as the ontology language. Obviously, the semantics of many ontology concepts become then more ambiguous.

Acknowledgements: Research presented in this paper was supported by the European Commission under contract ISTFP6-026978 X-Media.

\section{References}

[1] J. L. Herlocker, J. A. Konstan, A. Borchers and J. Riedl, "An algorithmic framework for performing collaborative filtering", Proceedings of SIGIR '99 Conference on Research and Development in Information Retrieval, ACM Press, New York, NY, 1999, pp. 230-237.

[2] M. Claypool, A. Gokhale, T. Miranda, P. Murnikov and M. Sartin, "Combining content-based and collaborative filters in an online newspaper", Proceedings of ACM SIGIR Workshop on Recommender Systems, August 1999.

[3] L.G. Terveen and W. Hillm, "Beyond Recommender Systems: Helping people help each other", In Carroll, J. (ed.) HCI in the New Millennium. Addison Wesley.

[4] G.-J. Houben and P. De Bra, "Automatic Hypermedia Generation for Ad Hoc Queries on Semi-Structured Data", Proceedings of the 5th ACM International Conference on Digital Libraries, 2000, pp. 240-241.

[5] O. Conlan, V. Wade, C. Bruen and M. Gargan, "Multimodel, Metadata Driven Approach to Adaptive Hypermedia Services for Personalized eLearning“, Lecture Notes in Computer Science, Vol. 2347, 2002, pp. 100-132.

[6] P. Brusilovsky and D.W. Cooper, "Domain, task, and user models for an adaptive hypermedia performance support system", Proceedings of the 7th international conference on Intelligent user interfaces, pp. 23 - 30.

[7] A. Aerts, B. Berden, B. de Lange, B. Rousseau, T. Santic, D. Smits, N. Stash, "Aha! the adaptive hypermedia architecture", Proceedings of the ACM Hypertext Conference, Nottingham, UK, 2003, pp. 81-84.

[8] J. Herlocker, J. Konstan and J. Riedl, "Explaining Collaborative Filtering Recommendations", ACM 2000 Conference on Computer Supported Cooperative Work.

[9] T. Gruber, "Toward principles for the design of ontologies used for knowledge sharing", Communications of the ACM 37(7), pp. 48-53.

[10] A. Pease, I. Niles and J. John, "The Suggested Upper Merged Ontology: A Large Ontology for the Semantic Web and its Applications", Working Notes of the AAAI-2002 Workshop on Ontologies and the Semantic Web, Edmonton, Canada, 2002.

[11] TOS Coalition, "OWL-S: Semantic Markup for Web Services”, W3C Member Submission, 2004.

[12] IEEE Learning Technology Standards Committee, "IEEE LOM Working Draft 6.1",

http://tsc.ieee.org/wg12/index.html, April 2001.

[13] M. Collett and F. Farance, "Draft standard for learning technology. Public and private information (PAPI) for learners (PAPI Learner)", Technical Report Version 7, IEEE LTSC (2000),

http://edutool. com/papi/drafts/07/papi/learner/.

[14] P. Dolog, R. Gavriloaie, W. Nejdl, J. Brase, ”Integrating Adaptive Hypermedia Techniques and Open RDFbased Environments", Proc. of 12th International World Wide Web Conference.

[15] R. Baumgartner, N. Henze and M. Herzog, "The Personal Publication Reader: Illustrating Web Data Extraction, Personalization and Reasoning for the Semantic Web". In European Semantic Web Conference ESWC 2005, 2005.

[16] B. Motik and U. Sattler, "Practical DL Reasoning over Large A-Boxes with KAON2", submitted for publication, http://kaon2.semanticweb.org/. 